DOI: $10.3901 / J M E .2020 .22 .001$

\title{
一种复杂结构件圆柱面扩散焊缝阵列 超声检测方法
}

\author{
李文涛 1,2 周正千 1,3
}

(1. 北京航空航天大学机械工程及自动化学院 北京 100083 ;

2. 兰州理工大学机电工程学院 兰州 730050;

3. 中国科学院声学研究所声场声信息国家重点实验室 北京 100190)

摘要: 航空发动机某高温合金盘构件采用盘体与叶片环进行焊接的方式制备，由于构件结构复杂，在检测圆柱形焊接面缺陷 时, 采用单晶超声检测换能器检测效率不高, 缺陷信号信噪比低。为提高该种结构件的检测能力, 研究圆柱面焊缝的阵列超 声自动检测方法, 分析面积型缺陷和声源的相对位置对缺陷反射声压和波型转换的影响，提出一种基于双阵列换能器的一发 一收检测方案。根据高温合金盘结构设计基于双阵列换能器的声束发射-接收方法和圆柱面焊缝全覆盖检测方案; 基于时域有 限差分方法建立高温合金盘的阵列超声声学响应仿真模型，对检测参数进行设计和优化。对含有人工缺陷的实际试样进行检 测试验, 结果表明所提出的双阵列超声换能器检测方法能有效检测到圆柱面焊缝区域 $\phi 2 \mathrm{~mm}$ 的当量缺陷。

关键词：阵列超声；圆柱面焊缝；无损检测；时域有限差分法

中图分类号: TB553

\section{Ultrasonic Array Detection Method on Cylindrical Surface Diffusion Weld of Complex Structural Parts}

\section{Wentao ${ }^{1,2}$ ZHOU Zhenggan ${ }^{1,3}$}

(1. School of Mechanical Engineering and Automation, Beihang University, Beijing 100083;

2. School of Mechanical and Electronical Engineering, Lanzhou University of Technology, Lanzhou 730050;

3. State Key Laboratory of Acoustics, Institute of Acoustic, Chinese Academy of Science, Beijing 100190)

\begin{abstract}
A superalloy disc component of aeroengine is fabricated by welding between disc and blade ring. Because of the complexity of component structure, the single element ultrasonic testing method has met same problems such as low detection efficiency and low signal-to-noise ratio of defect signals. In order to improve the detection ability of this kind of structure, the automatic ultrasonic array detection method of the cylindrical weld is studied. Firstly, the effect of relative positions of the planar defect and the acoustic source on the defect refection acoustic pressure is analyzed. The wave conversion conditions of different reflected acoustic waves are analyzed and the pitch and catch method based on dual ultrasonic array transducers is put forward. Then, according to the structural characteristics of the specimen, an acoustic beam transmitting-receiving method and a full coverage inspection scheme for the cylindrical weld are designed. A response simulation model of the ultrasonic array is established based on the finite-difference time-domain (FDTD) method and the detection parameters are designed and optimized. Finally, the detection experiment is carried out on the actual specimen with prefabricated defects, and the results show that the proposed dual ultrasonic array transducer detection method can effectively detect the prefabricated defects with diameter of $2 \mathrm{~mm}$ in the cylindrical weld area.
\end{abstract}

Key words: ultrasonic array; cylindrical weld; non-destructive testing(NDT); finite difference time domain method

* 声场声信息国家重点实验室资助项目(SKLA201810)。20191228 收到初稿, 20200828 收到修改稿 


\section{0 前言}

扩散焊技术具有焊接后内应力和变形小等优 势, 现已应用于航空发动机领域中高温合金盘结构 件的制造, 可实现圆柱形盘体和外部叶片环的整体 连接。此类结构件在焊接过程中受焊接表面粗糙度 和杂质等因素的影响, 圆柱面焊接界面会产生未焊 合的面积型缺陷, 严重威胁航空发动机工作的安 全 ${ }^{[1-2]}$ 。然而, 不同于孔洞、气孔等体积型缺陷, 面 积型缺陷与超声换能器的相对位置会很大程度上影 响其检测结果 ${ }^{[3]}$ 。当入射声束方向与面积型缺陷的 取向不垂直时, 缺陷回波信号可能难以被发射换能 器接收 ${ }^{[4]}$ 。由于高温合金盘结构复杂、材料特殊, 目前采用单晶聚焦超声换能器检测时, 声束声程大、 扩散和衰减严重, 使得缺陷检测分辨率不高 ${ }^{[5]}$, 可 以检测不小于 $\phi 3.2 \mathrm{~mm}$ 的当量缺陷, 且检测效率不 高。由于该结构件在实际使用中的受力情况较为复 杂, 目前检测方法的检测能力尚不能完全满足其在 高强度环境中的使用要求。因此, 为了提高该种结 构件的检测能力, 亟需一种可靠、有效的新型无损 检测方法对高温合金盘圆柱面焊缝缺陷进行全覆盖 检测。

阵列超声检测技术具有检测速度快, 缺陷分 辨率高和检测精确性高等优点, 可通过控制阵列 超声换能器中各独立压电晶片发射/接收声波的 延迟时间来控制声束的聚焦和偏转 ${ }^{[6]}$ 。阵列超声 无损检测方法目前已被广泛应用于航空发动机叶 盘叶片、复合材料 L 型构件等复杂结构的无损检 测中, 且取得了较好的检测结果 ${ }^{[7-9]}$ 。然而, 圆柱 面焊缝界面产生的缺陷主要为未焊合缺陷, 受高 温合金盘的结构限制, 阵列超声换能器的声束无 法垂直入射在焊缝界面, 导致采用单一的阵列超 声换能器难以接收到有效的缺陷回波信号, 且合 成声束不能有效覆盖整个焊缝区域。为此, 需要 针对高温合金盘的结构和缺陷分布特点研究新的 阵列超声检测和成像方法。

本文在研究阵列超声检测技术基础上基于双阵 列超声换能器提出了一种一发一收的阵列超声检测 方法, 针对高温合金盘扩散焊缝的检测设计了相应 的合成声束发射-接收算法, 利用数值仿真方法分析 和验证了检测方案的正确性, 搭建了双阵列超声换 能器的检测软硬件系统, 对预制缺陷的高温合金盘 试样进行了扫查试验, 检测能力和检测效率相对单 晶超声换能器均有较大幅度提高。

\section{1 检测方案设计}

\section{1 声束发射接收方法建立}

扩散焊缝界面的未焊合缺陷与检测声源的相对 位置对检测结果有很大的影响, 因此, 首先研究未 焊合面积型缺陷与声源的相对位置对回波信号幅值 的影响(图 1), 假设主声束中心轴线的偏转角度为 $\theta$, 面积型缺陷的水平倾斜角为 $\tau$, 可以得到声束在 缺陷中心的入射角为 $\tau-\theta$, 则反射声波在换能器中 心点处的声压可以表示为 ${ }^{[10]}$

$$
P_{t}=r_{\mathrm{LL}} p_{0} D_{\mathrm{L}}^{2}(\theta) \cos (\theta-\tau) \frac{\sin \left[\boldsymbol{k}_{\mathrm{L}} c \sin (\theta-\tau)\right]}{\boldsymbol{k}_{\mathrm{L}} c \sin (\theta-\tau)}
$$

式中, $r_{\mathrm{LL}}$ 是声波在空气-试件界面的声压反射系数; $p_{0}$ 是垂直入射时的缺陷回波声压; $D_{\mathrm{L}}$ 是声束的指向 性系数; $\boldsymbol{k}_{\mathrm{L}}=2 \pi / \lambda$ 为波矢量, 其中 $\lambda$ 是声波波长。 当 $\theta \approx \tau$ 时, 缺陷的反射声压最大, 此时主声束轴线 与缺陷平面垂直, 换能器接收到的缺陷回波声压最 高; 然而, 当 $\tau-\theta>0$ 时, 即缺陷的水平倾斜角大于 主声束中心轴线的偏斜角时, 换能器接收不到主声 束与缺陷作用后的反射声波，且面积型缺陷的尖端 衍射信号很弱，难以用于缺陷评判。可以发现，面 积型缺陷的检测结果受缺陷取向及其与声源相对位 置的共同影响，在设计超声检测方案时要对试样结 构和所采用的声束发射接收方法进行综合考虑。

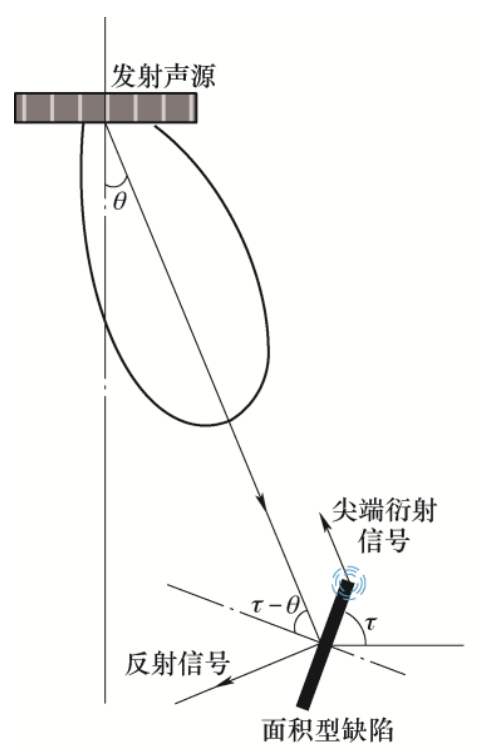

图 1 面积型缺陷与声源轴线偏离时的反射示意图

高温合金盘是一种由外部的叶片环和内部浴轮 盘体焊接而成的复杂整体焊接结构，无法采用阵列 超声换能器对焊接界面进行直接的垂直入射检测。 图 2 所示为高温合金盘整体结构和采用双阵列超声 
换能器检测的布置示意图, 当阵列超声换能器 $-A$ 发 射时, 通过阵列超声换能器 $-B$ 可以有效地接收到缺 陷反射信号。但是, 由于阵列超声换能器与检测焊 缝的水平距离很近, 且焊缝沿坚直方向分布, 由式 (1)可知, 此时缺陷的反射声压较弱, 若直接利用声 束偏转进行检测, 所得到的焊缝界面缺陷信号将十 分微弱或被其他信号所淹没。此外, 由于高温合金 盘坚直焊缝贯穿于整个试样, 要实现整个焊缝区域 面积型缺陷的检测还需要对声束偏转聚焦参数进行 设计。因此, 将以高温合金盘整体的圆柱面焊缝为 检测对象, 进一步设计基于双阵列超声换能器的声 束控制方案和成像方法, 实现当前焊接界面的全覆 盖检测。

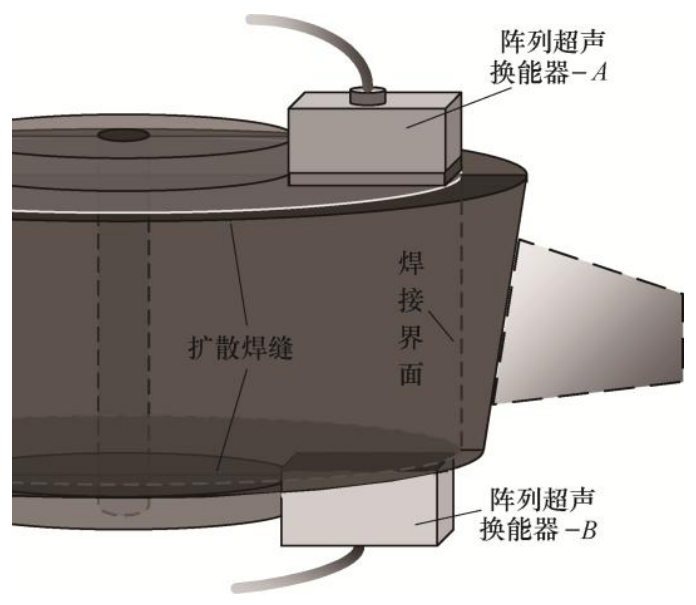

图 2 双阵列超声换能器检测高温合金盘扩散焊缝的示意图

为了避免阵列超声换能器近场和较大声束偏转 角度对检测结果的影响 ${ }^{[11]}$, 首先利用特定的聚焦延 迟法则激励阵列超声换能器 $-A$ 进行发射, 采用阵列 超声换能器 $-B$ 进行接收的方式对靠近换能器 $-B$ 的 下半部分焊缝进行检测, 然后交换发射-接收换能器 和相应的聚焦法则实现上半部分焊缝的检测(图 3)。 假设两个换能器的阵元数目均为 $N / 2$ 个, 以阵列超 声换能器 $-A$ 为例, 其合成声束中心的偏转角度 $\theta_{i}$ 范 围为

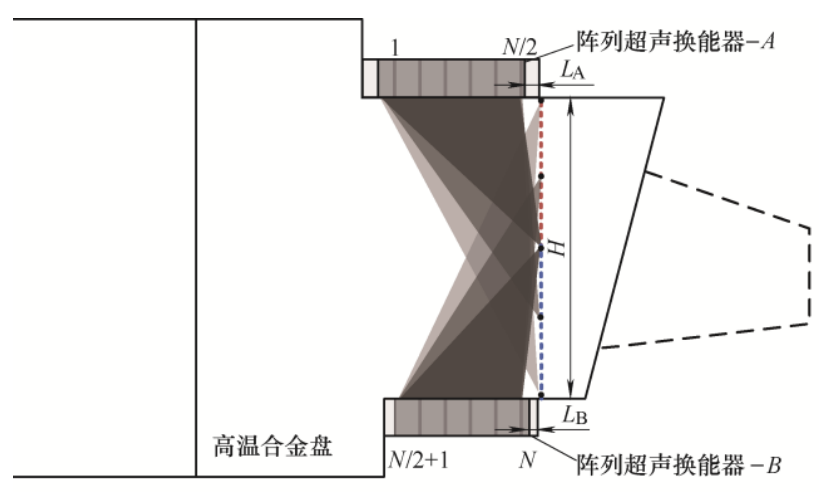

图 3 高温合金盘焊缝的一发一收阵列超声检测方案

$$
\arctan \frac{N \times \frac{d}{4}+L_{A}}{H} \leqslant \theta_{i} \leqslant \arctan \frac{N \times \frac{d}{4}+L_{A}}{\frac{H}{2}}
$$

式中, $d$ 为阵元间距; $L_{\mathrm{A}}$ 为阵列超声换能器 $-A$ 第 $N / 2$ 个阵元与焊缝的水平距离; $H$ 为焊缝的坚直高度。

由于扩散焊缝中面积型缺陷的水平倾斜角 $\tau$ 为 固定的 $90^{\circ}$, 进而可计算出不同位置缺陷的声波入 射角度。然而, 根据入射波的类型和入射角度的不 同, 经界面反射后会产生不同的波型转换, 在检测 面积型缺陷时会影响缺陷信号的评判, 因此还需要 进一步确定聚焦声波的类型。图 4a、4b 分别为纵波 和横波声束以一定角度入射时会出现的波型转换示 意图。图 4a 所示为纵波以角度 $\theta_{\mathrm{L}}$ 入射时反射声波 的波型转换，当入射角度 $\theta_{\mathrm{L}}$ 逐渐增大时，反射声波 将同时包含纵波和横波; 图 $4 \mathrm{~b}$ 所示为当入射声波为 横波时, 随着入射角度 $\theta_{\mathrm{s}}$ 的增大, 纵波信号将会消 失只存在反射横波。结合式(2)和高温合金盘几何尺 寸, 得到检测方案中声波的最小入射角度为 $65.3^{\circ}$, 如果采用纵波聚焦, 与缺陷作用后的大部分能量将 转换为反射横波且同时存在反射纵波，导致缺陷反 射信号成分复杂, 能量较弱 ${ }^{[12-13]}$ 。因此, 采用聚焦 横波进行入射检测时, 可使得面积型缺陷的反射信 号为单一成分的横波信号。

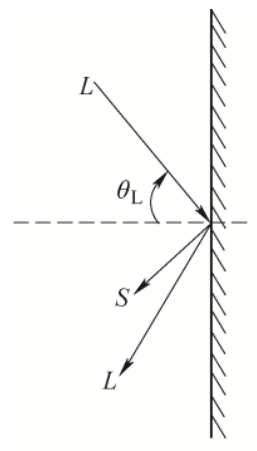

(a) 纵波入射

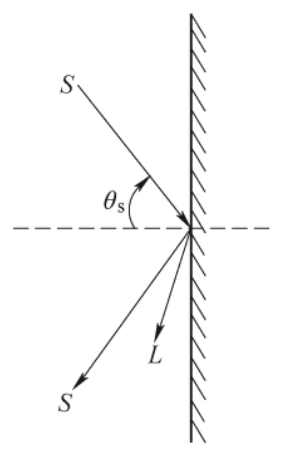

(b) 横波入射
图 4 不同波型入射情况下反射声波的波型转换

\section{2 聚焦延迟时间计算}

不同于常规的阵列超声扇形扫查方式, 在本试 样的检测中, 阵列超声换能器的合成声束在聚焦偏 转角度变化的同时还需要改变当前通道的聚焦点深 度 ${ }^{[14]}$, 如图 5 所示, 以焊缝与试样下表面交点 $O$ 为 坐标原点, 假设阵列超声换能器的阵元宽度为 $w$, 坚直焊缝的高度为 $H, c_{\mathrm{s}}$ 是试件中的横波声速, 声 束聚焦到焊缝 $P\left(0, z_{\mathrm{p}}\right)$ 点时, 由费马原理可得第 $i$ 个 阵元到达聚焦点 $P$ 的声传播时间

$$
T_{i}=\min _{z_{\mathrm{p}} \leqslant \frac{H}{2}} \sqrt{\frac{x_{i}^{2}+\left(H-z_{\mathrm{p}}\right)}{c_{\mathrm{S}}^{2}}}
$$


式中, $x_{i}=-L_{\mathrm{A}}-\left[\left(\frac{N}{2}-i\right) \times d+\frac{w}{2}\right]$, 其包含的值均为

已知量, $T_{i}$ 的值可根据特定聚焦点在 $Z$ 轴对应的 $z_{\mathrm{p}}$ 值求出。

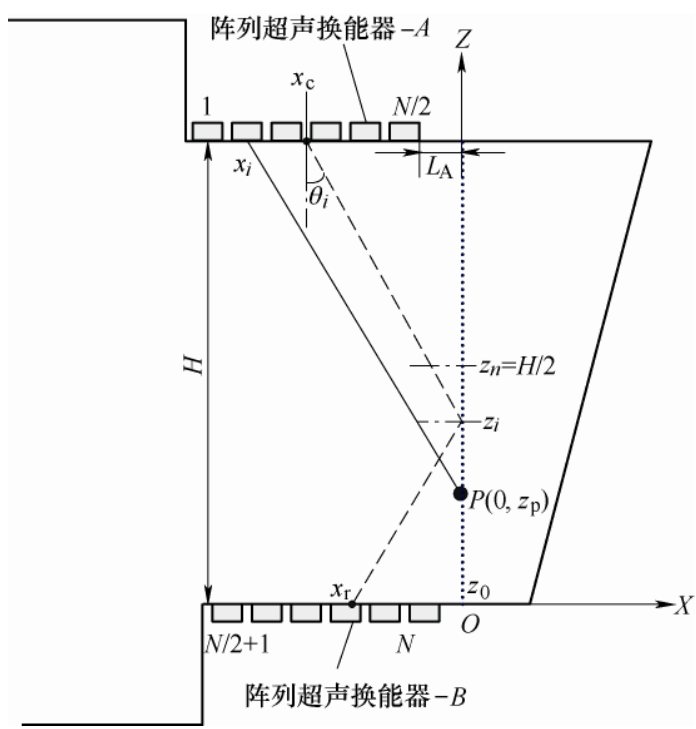

图 5 扩散焊缝区域检测的延迟时间计算

因此, 针对焊缝界面的某一固定聚焦点, 重复 式(3)可计算出当前通道中所有激发阵元发出的声 波传播到该聚焦点的时间，将最大传播时间定义为 $T_{\max }$, 则阵元 $i$ 所对应的延迟时间 ${ }^{[15]}$

$$
\Delta T_{i}=T_{\text {max }}-T_{i}
$$

当阵列超声换能器 $-A$ 作为发射换能器时, 将靠 近阵列超声换能器 $-B$ 的下半部分焊缝离散为一定数 量的聚焦点 $z_{0} \sim z_{n}$, 再分别对 $n+1$ 个特定的聚焦延 迟法则进行设计。当阵列超声换能器 $-B$ 发射时, 根 据几何结构的对称性可直接应用阵列超声换能器- $A$ 的聚焦法则对上半部分焊缝进行检测。然而, 受高 温合金盘外围侧壁的影响, 阵列超声换能器还会接 收到除缺陷反射信号以外的侧壁反射信号, 且焊缝 不同位置缺陷信号传播到接收换能器的总声程有所 差异, 即如图 5 所示的 $\left|x_{\mathrm{c}} z_{i}\right|+\left|z_{i} x_{\mathrm{r}}\right|$ 。因此在确定 离散聚焦点后, 还需要计算声波传播到各离散点再 被反射到接收换能器的总传播时间, 进而针对每一 个通道设计特定的闸门参数, 准确提取不同位置缺 陷的反射信号。假设高温合金盘试件为各向同性介 质, 横波声速随传播角度的变化保持不变, $x_{\mathrm{c}}$ 为换 能器中心点的横坐标, 则阵列超声换能器 $-A$ 的合成 声束传播至聚焦点 $z_{i}$ 再被缺陷反射后到达接收换能 器 $-B$ 的传播时间为

$$
t_{i}=\frac{\sqrt{\left.\left(H-z_{i}\right)^{2}+x_{\mathrm{c}}^{2}\right)}+\sqrt{z_{i}^{2}+\left(z_{i} \times \frac{x_{\mathrm{c}}}{H-z_{i}}\right)^{2}}}{c_{\mathrm{s}}}
$$

\section{2 缺陷响应仿真及检测试验}

\section{1 有限差分法的数值仿真验证}

高温合金盘主体部分的材料为镍基高温粉 末合金, 圆柱面焊缝的高度 $H$ 为 $36.6 \mathrm{~mm}$, 焊缝 位置处直径为 $\phi 125 \mathrm{~mm}$, 根据试件声速、最小检 测缺陷尺寸和换能器位置参数等条件, 设计两个 阵列超声换能器的中心频率、阵元数量、阵元间 距等主要参数, 并利用声场指向性和轴向声压分 布情况对设计参数进行评价，针对高温合金盘试 样所设计的双阵列超声换能器的主要检测参数 如表 1 所示。

\section{表 1 阵列超声换能器参数}

\begin{tabular}{lc}
\hline \multicolumn{1}{c}{ 参数 } & 数值 \\
\hline 中心频率 $/ \mathrm{MHz}$ & 5 \\
阵元数量 & 32 \\
阵元间距 $/ \mathrm{mm}$ & 0.4 \\
阵元宽度 $/ \mathrm{mm}$ & 0.3 \\
偏转角度 $/\left(^{\circ}\right)$ & $13.1 \sim 24.7$ \\
聚焦深度 $/ \mathrm{mm}$ & $18.3 \sim 36.6$ \\
\hline
\end{tabular}

为了验证和优化整体检测方案的正确性，基于 时域有限差分(FDTD)方法建立了高温合金盘焊 缝面积型缺陷检测的二维仿真模型(图 6)。仿真模 型的网格尺寸大小为 $0.02 \mathrm{~mm}$ ，仿真时间间隔 $1 \mathrm{~ns}$, 仿真总时长 $25 \mu \mathrm{s}$ 。阵元激励载荷为压力载 荷, 垂直于阵列超声换能器阵元的排列方 向 $^{[16]}$ 。 加载的激励信号为 3 周期的正弦信号, 中心频率 为 $5 \mathrm{MHz}$ 。同时, 在焊缝中心深度 $18 \mathrm{~mm}$ 处切割 一个长 $1 \mathrm{~mm}$ 、宽 $0.2 \mathrm{~mm}$ 的长矩形来模拟面积型 缺陷。高温合金盘材料的纵波波速为 $5980 \mathrm{~m} / \mathrm{s}$, 横波波速为 $3138 \mathrm{~m} / \mathrm{s}$ 。



图 6 高温合金盘阵列超声检测声学模型 
根据所设计的双阵列超声换能器聚焦横波 检测方案, 阵列超声合成声束的传播及其与缺陷 的作用结果如图 7 所示。在图 7a 所示的初始时 刻, 根据所设计的聚焦延迟法则, 发射换能器- $A$ 所激励的声波包含聚焦横波 $A$ 和纵波 $C$ 。当聚焦 横波作用在缺陷上时, 聚焦横波 $A$ 中部分声波斜 入射到缺陷表面产生反射横波 $B$ (图 7b)。在图 7c 中, 缺陷反射横波 $B$ 和侧壁的反射信号将顺利传 播至贴合于高温合金盘另一侧表面的阵列超声 换能器 $-B$ 。

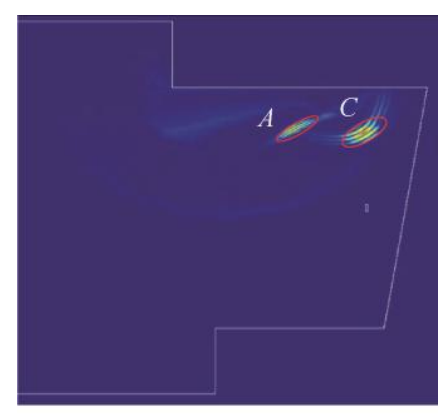

(a) 时刻一

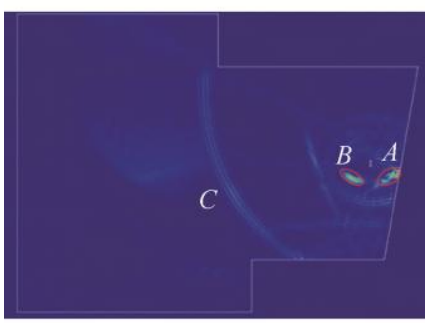

(b) 时刻二



(c) 时刻三

图 7 阵列超声聚焦横波的传播过程与反射结果

分别建立无缺陷的仿真模型和包含有面积型缺 陷的仿真模型, 对每个接收阵元所接收到的信号进 行相应的相位延迟处理, 提取并绘制换能器所接收 到的超声信号, 该通道对应的缺陷信号传播到接收 换能器的时间可根据式(5)确定。图 8 所示为无缺陷 模型和含有缺陷模型中换能器所接收到的 $\mathrm{A}$ 型信 号。从仿真结果可以看出, 该检测方法对面积型缺 陷具有较高的检测信噪比和灵敏度, 验证了检测方 案和检测参数的正确性。

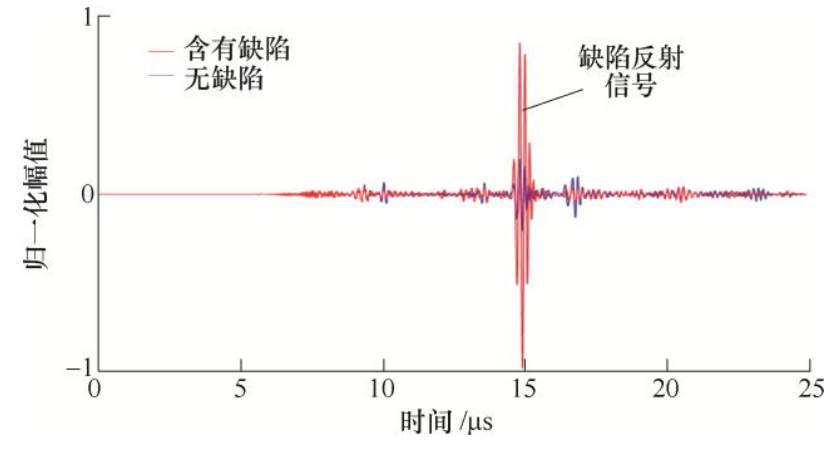

图 8 一发一收阵列超声检测方案的缺陷响应仿真结果

\section{2 检测试验及应用}

为便于加工，预制缺陷的检测试样为不包含叶 片的盘体部分，材料采用与高温合金声速接近的碳 素钢制成。在高温合金盘试样外壁距离底面 $8 \mathrm{~mm}$ 处加工有直径为 $2 \mathrm{~mm}$ 的平底孔, 孔底刚好位于焊 接界面用来模拟焊缝中的面积型缺陷, 试样实物如 图 9 所示。

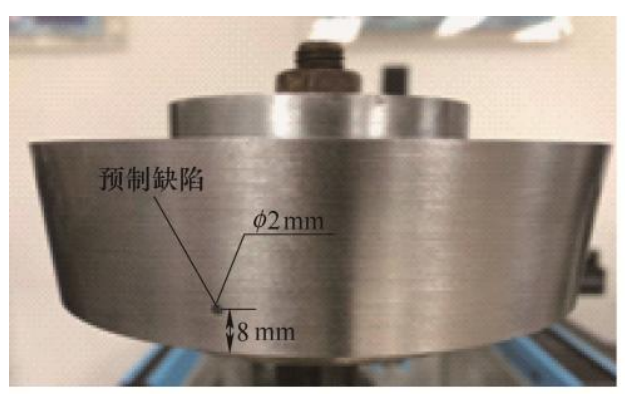

图 9 高温合金盘试样实物图

根据所设计的检测方案建立基于双阵列超声换 能器的检测系统, 其整体组成如图 10 所示, 两个阵 列超声换能器通过分线器连接到阵列超声板卡, 扫 描工装可在固定阵列超声换能器的同时利用底部电 机带动试样旋转, 通过计算机控制阵列超声板卡和 扫描工装实现自动检测, 并对采集到的数据进行处 理, 直观显示整个扩散焊接界面的检测结果 ${ }^{[17]}$ 。

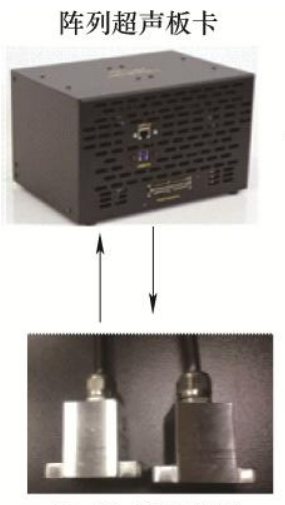

阵列超声换能器

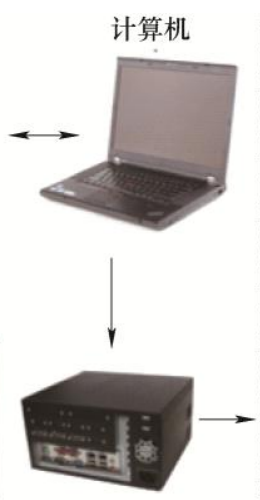

电机控制箱



扫描工装
图 10 高温合金盘阵列超声自动检测系统 
设置沿焊缝深度方向的聚焦点离散间隔为 $0.5 \mathrm{~mm}$, 每个通道的声波传播路径和时间是确定的, 利用不同通道所对应的闸门对焊缝不同位置的缺陷 反射信号进行提取, 通过改变双阵列超声换能器的 声束发射-接收算法能在不移动换能器的情况下完 成整条焊缝的检测, 配合旋转扫查装置可实现整个 高温合金盘扩散焊焊缝的 C 扫描成像。此外, 利用 计算机图形处理器并行算法和 Open GL 三维图像映 射等方法, 实现了高温合金盘试样整体焊缝面积型 缺陷的自动 $\mathrm{C}$ 扫检测和实时三维显示, 检测结果如 图 11 所示。

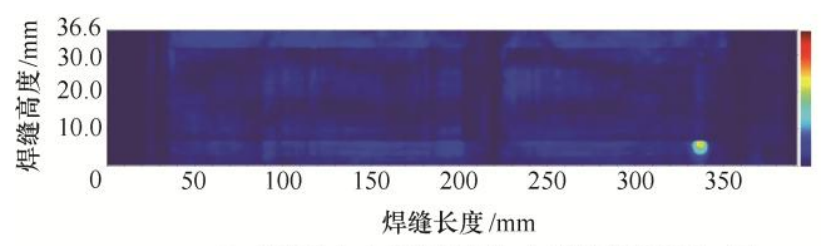

(a) 高温合金盘扩散焊缝的C扫描检测结果展开图

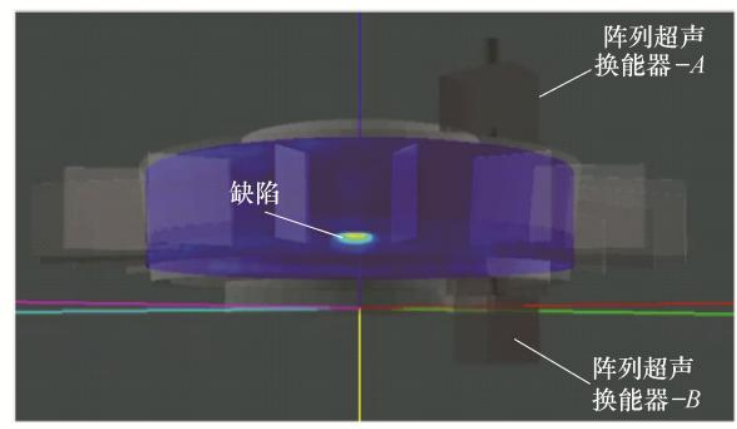

(b) 高温合金盘试样检测结果的三维显示

图 11 高温合金盘试样的一发一收阵列超声检测结果

检测试验结果能直观地观测高温合金盘焊缝区 域缺陷分布情况, 通过对比验证, $\mathrm{C}$ 扫描检测结果 中缺陷的位置和尺寸与试样中预制的缺陷有很好的 对应性, 相对于常规单晶超声换能器的检测方法, 双阵列超声换能器一发一收的检测方案具有声束路 径短、检测方式灵活等优势, 对于圆柱面焊缝中未 焊合缺陷具有很高的灵敏度。

\section{3 结论}

（1）针对高温合金盘圆柱面焊缝区域缺陷的 检测, 提出的基于双阵列超声换能器的一发一收 检测方法具有检测灵活性高、声束覆盖范围广等 特点, 可进一步推广应用于其他复杂结构和特殊 构件的检测。

(2) 利用 FDTD 方法建立的阵列超声数值仿真 模型能用于对阵列超声换能器参数、延迟时间和聚 焦波型等检测参数的验证, 对采用多个阵列超声换
能器的检测方案设计和优化具有重要意义。

(3) 结合机械扫描系统实现了高温合金盘试样 的自动化检测, 可以检测出 $\phi 2 \mathrm{~mm}$ 的当量缺陷, 利 用图像映射算法得到了圆柱面焊缝 C 扫描结果的三 维显示。检测结果表明, 采用所设计的阵列超声检 测和成像方法比采用单晶超声换能器的检测能力提 高了 1.5 倍以上, 检测效率提高了数十倍。

\section{参 考 文 献}

[1] DELRUE S, NISHIO K, TABATABAEIPOUR M, et al.

Applying a nonlinear, pitch-catch, ultrasonic technique for the detection of kissing bonds in friction stir welds[J]. Ultrasonics, 2016, 68: 71-79.

[2] 刘松平, 郭恩明, 谢凯文, 等. 钛合金扩散焊中紧贴型 缺陷的超声波检测 [J]. 无损检测，2004，26(2)：62-65. LIU Songping, GUO Enming, XIE Kaiwen, et al. Evaluation of kiss defect in diffusion welding of titanium alloy by ultrasonic pulse-echoes[J]. Nondestructive Testing, 2004, 26(2): 62-65.

[3] JONATHAN A, ANTHONY C, JACK P, et al. Nonlinear non-collinear ultrasonic detection and characterisation of kissing bonds[J]. NDT\&E International, 2018, 99 : 105-116.

[4] 张驰, 奕亦琳, 罗志伟, 等. 扩散焊接头缺陷超声 C 扫描检测能力分析 [J]. 焊接学报, 2016, 37(9): 83-86, 90, 132.

ZHANG Chi, LUAN Yilin, LUO Zhiwei, et al. Analysis of ultrasonic C-scan detectability on diffusion bonding joint[J]. Transactions of the China Welding Institution, 2016, 37(9): 83-86, 90, 132.

[5] 刘松平, 李乐刚, 刘菲菲, 等. 大厚度扩散焊 NLU 成 像检测技术[J]. 航空制造技术，2017(5)：34-37， 48.

LIU Songping, LI Legang, LIU Feifei, et al. Evaluation of thick diffusion bonds by using NLU imaging method[J]. Aeronautical Manufacturing Technology, 2017(5): 34-37, 48.

[6] LUPIEN V, HASSAN W, DUMAS P. Improved titanium billet inspection sensitivity through optimized phased array design[C]// 32nd Annual Review of Process in Quantitative Nondestructive Evaluation, July 31-August 5, 2005. Brunswick: American Institute of Physics, 2006, 853-860.

[7] 陶博伟. 航空发动机高温合金盘相控阵检测系统研制 [D]. 南昌: 南昌航空航天大学, 2013.

TAO Bowei. Research of superalloy turbine disk by 
phased array testing system[D]. Nanchang: Nanchang Hangkong University, 2013.

[8] XU N, ZHOU Z G. Numerical simulation and experiment for inspection of corner-shaped components using ultrasonic phased array[J]. NDT\&E International, 2014, 63: 28-34.

[9] LI W T, ZHOU Z G, LI Y. Inspection of butt welds for complex surface parts using ultrasonic phased array[J]. Ultrasonics, 2019(96): 75-82.

[10] 赛鹏, 王佐森, 张建磊, 等. 焊缝根部及近根部垂直面 积型缺陷的超声检测 [J]. 无损检测, 2016, 38(9): 53-56. SAI Peng, WANG Zuosen, ZHANG Jianlei, et al. Ultrasonic examination for planar defects perpendicular to examination surface at or near the weld $\operatorname{root}[\mathrm{J}]$. Nondestructive Testing, 2016, 38(9): 53-56.

[11] 万敏, 王海涛, 程继隆, 等. 超声相控阵声束控制特 性分析[J]. 无损检测，2009，31(11)：859-861.

WAN Min, WANG Haitao, CHENG Jilong, et al. The analysis on the features of the propagating waves control of the ultrasonic phased array[J]. Nondestructive Testing, 2009, 31(11): 859-861.

[12] DRINKWATER B W, WILCOX P D. Ultrasonic arrays for non-destructive evaluation: A review[J]. NDT \& E International, 2006, 39(7): 525-541.

[13] ROSE J L. Ultrasonic waves in solid media[M].
Cambridge: Cambridge University Press, 1999.

[14] SCHMERR L W. Fundamentals of ultrasonic phased arrays[M]. New York: Springer International Press, 2015.

[15] AZAR L, SHI Y, WOOH S C. Beam focusing behavior of linear phased arrays[J]. NDT\&E International, 2000, 33(3): $189-198$

[16] BLOXHAM H, VELICHKO A, WILCOX P D, et al. Combining simulated and experimental data to simulate ultrasonic array data from defects in materials with high structural noise $[\mathrm{J}]$. Transactions on Ultrasonic Ferroelectrics and Frequency Control, 2016(63) : 2198-2206.

[17] 王常胥, 钢铁, 于朋, 等. 一种铝合金薄板搅拌摩擦焊 焊缝的超声快速检测方法 [J]. 机械工程学报, 2015, 51(2): 7-13.

WANG Changxi, GANG Tie, YU Peng, et al. Ultrasonic method for rapid detection of the aluminum friction stirwelding defects[J]. Journal of Mechanical Engineering, 2015, 51(2): 7-13.

作者简介: 李文涛, 男, 1989年出生, 博士研究生。主要研究方向为现 代超声检测技术。

E-mail: liwentao@buaa.edu.cn

周正干(通信作者), 男, 1967年出生, 博士, 教授。主要研究方向为超 声无损检测和计算机测控技术等。

E-mail: zzhenggan@buaa.edu.cn 